Historic, Archive Document

Do not assume content reflects current scientific knowledge, policies, or practices. 



\section{THE CONARD-PYLE COMPANY}

\section{" ROBERT PYLE, President}

\section{West Grove, Pennsylvania}

Terms and conditions as in 1927 Spring Trade List: Prices f. o. b. West, Grove and sub-

save money by getting 25 of a kind at the 100 rate; 250 at the 1000 rate, Inquiries solicited.

\section{Make the Most of Your Money this Spring} with

\section{$\rightarrow \star \operatorname{ROSES}$}

Vigorous plants skillfully stored by modern methods. Every root carefully protected. You can get better results with these Roses! Hybrid Teas and Teas 2 year No. 1 budded, dormant plants

210 Betty. HT. Coppery rose, overspread with yellow............. $\$ 4.50 \quad \$ 40.00$ 200 Charles K. Douglas. HT. Striking crimson-scarlet.............4.50 $\begin{array}{r}\$ 40.00 \\ 40.00\end{array}$ 400 Florence Pemberton. HT. Light pink; beautiful, well-pointed buds 4.00 500 George Dickson. HP. Immense dark red velvety bloom. Never "blues" 4.00 35.00 70 Gorgeous. HT. Light yellow, overspread with copper and orange..... 4 50 40.00 150 Grange Colombe. HT. Creamy white with salmon center...... $500 \mathrm{H}$. V. Machin. HT Massive crimson buds Particularly beautiful $4.00 \quad 35.00$ 35 Independence Day HT Flaming yellow buds Hicula 35 Independence Day. HT. Flaming yellow buds. Herriot type..... 5.50 50.00 190 Killarney Queen. HT. Like Killarney, but fuller and stronger. . . . . 4.00 45.00 260 Lady Alice Stanley. HT. Outside coral-rose; inside pale pink. . . 4.00 35.00 30 Lady Ursula. HT. Light pink. One of the best blooming Roses. . . . . 4.00 45.00 600 La Tosca. HT. Bright silvery pink. A fine decorative Rose........ $4.00 \quad 35.00$ 85 Louise C. Breslau. HT. Reddish orange. A very attractive Rose..... $4.50 \quad 40.00$ 175 Mary, Countess of Ilchester. HT. Deep rose-pink............ $4.00 \quad 35.00$ 100 Mme. Caroline Testout. HT. Satiny rose. One of the best-liked Roses $4.00 \quad 35.00$ 100 Mme. Gustave Metz. HT. "Frau Karl Smid". 750 Miss Cynthia Forde. HT. Sparkling pink. One of the best . . . . . 4.00 485 Miss Lolita Armour. HT. Creamy copper with reddish orange tinge... 4.50 40.00 120 Mrs. A. R. Waddell. HT. Yellowish buds, opening pink and apricot 4.0075 180 Mrs. Coore Shawyer. HT. Clear, brilliant rose-pink 180 . George Shawyer. HT. Clear, briliant rose-pink............ 4.00 300 Mins. Light pink inside of petals, outside deep pink $5.50 \quad 50.00$ 300 Mrs. W. C. Miller. HT. Light pink. An all-round satisfactory variety $4.00 \quad 35.00$ 800 Padre. HT. Copper-scarlet. Bound to be popular........... 5.50 50.00

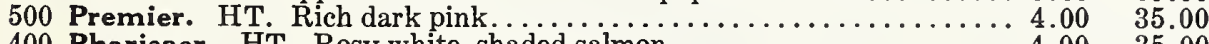

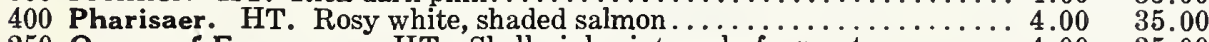
250 Queen of Fragrance. HT. Shell-pink; intensely fragrant........ $4.00 \quad 35.00$ 300 Prince de Bulgarie. HT. Flesh, tinted salmon and saffron.......... 5.00 45.00 300 Senateur Mascuraud. HT. Light yellow................ $5.00 \quad 40.00$ 200 Souvenir ciu President Carno.. HT. Rosy white. Fine to cut............ $4.00 \quad 55.00$ 1000 Wm. F. Dreer. HT. Shell-pink, yellow at base. Color fades less than many of the yellow varieties....................... 4.50

No. 2 HYBRID TEA ROSES FOR POTTING UP

2 year dormant plants. Prices $\$ 25$ per 100, except as otherwise noted. 25 Betty. Coppery rose. 160 Louise C. Breslau. Reddish orange. 125 Charles K. Douglas. Crimson scarlet. $200 \mathrm{Mme}$. Caroline Testout. Satiny rose.

50 Columbia. Rose pink. 70 Miss Cynthia Forde. Sparkling pink.

100 Florence Pemberton. Light pink.

50 George C. Waud. Light red.

75 Gruss an Teplitz. Brilliant crimson.

130 H. V. Machin. Crimson.

60 Miss Lolita Armour. Orange.

90 Mrs. Henry Morse. Light pink (\$35 per 100).

50 Mrs. S. K. Rindge. Buttercup yellow.

90 Padre. Copper scarlet (\$35 per 100).

independence Day. Flang
per 100 Souv. de Georges Pernet. Brilliant pink 60 Killarney Queen. Sprightly pink.

60 Lady Ursula. Light pink. $(\$ 35$ per 100$)$.

$150 \mathrm{Wm}$. F. Dreer. Shell pink and yellow.

\section{HARDY CLIMRING ROSES}

These are offered as two year, first grade, dormant plants, and will be shipped with tops trimmed in the usual fashion, except that patrons who require all branches uncut are asked to specify when ordering and remit $\$ 2$ extra per 100 .

250 Alida Lovett. HW. Similar to Dr. Van Fleet................ $\$ 4.00 \quad \$ 35.00$ 2900 American Pillar. HW. Brilliant crimson-pink. Becoming popular. . 3.00 20.00 250 Aviateur Bleriot. HW. One of the yellowest Climbers . . . . . . $4.00,25.00$

475 Bess Lovett. HW. "Better than Climbing American Beauty". 4.0035 .00

475 Bess Lort. HW "A white Taum"

400 Bonnie Prince. HW. "A white Tausendschon" $H \ldots \ldots \ldots \ldots \ldots .4 .0035 .00$

400 Christine Wright. HW. Wild-rose-pink. Similar to Dr. Van Fleet. . 4.00 30.00

600 Coronation. HW. Similar to Excelsa ............................. 3. $00 \quad 25.00$

4500 Dorothy Perkins. HW. Too popular to need description.......... 3.00 20.00

1600 Dr. Huey. HW. Unique new variety. Deep crimson-maroon ......., 6.00 50.00

8000 Dr.Van Fleet. HW. Pure apple-blossom-pink. "Thebest of all Climbers" $3.50 \quad 25.00$

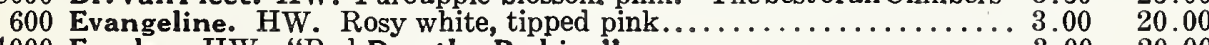

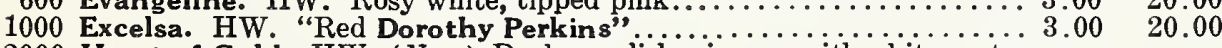

2000 Heart of Gold. HW. (New.) Dark purplish crimson, with white center

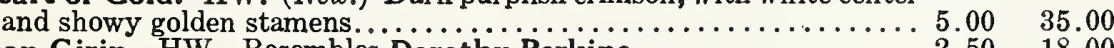

300 Jean Girin. HW. Resembles Dorothy Perkins................. 2.50 18.00

700 Lady Gay. HW. Considered the same as Dorothy Perkins ........ 3.00 20.00

500 Mary Wallace. HW. Very bright pink. One of Dr. Van Fleet's iast and

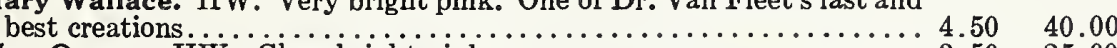

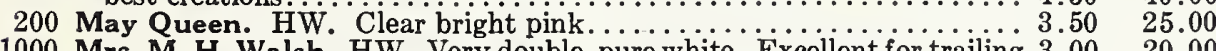

1000 Mrs. M. H. Walsh. HW. Very double, pure white. Excellent for trailing $3.00 \quad 20.00$

1000 Prof. C. S. Sargent. HW. One of the hardiest yellow Climbers; used

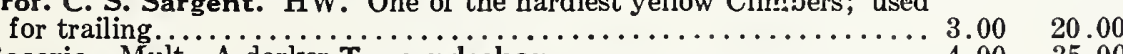

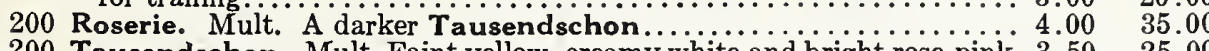

200 Tausendschon. Mult. Faint yellow, creamy white and bright rose-pink $3.50 \quad 25.00$ 1000 White Dorothy. HW. Creamy white. "White Dorothy Perkins" .. 3.00 20.00

BROADEN YOUR BUSINESS WITH HUGONIS

"Golden Rose of China"

(For descriplion see next page)

Vigorous dormant plants, field-grown (O. R.)

$110 \quad 25,50,100250,500,1,000$ up

Extra 2-year. 


\section{if ROSES-Continued}

\section{HYBRID PERPETUALS AND MISCELLANEOUS ROSES}

Two year, frst grade dormant plants.

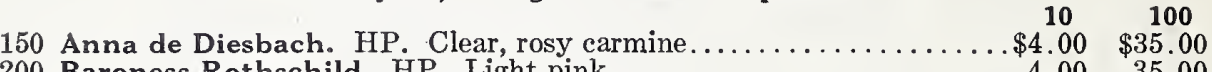

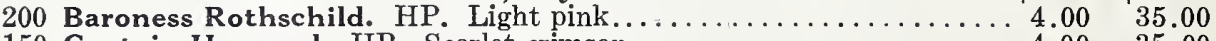

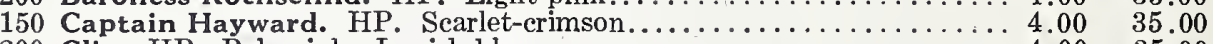

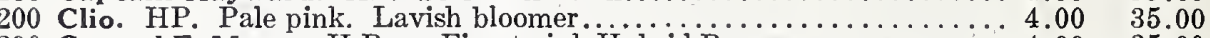

300 Conrad F. Meyer. H.Rug. Finest pink Hybrid Rugosa . . . . . . . . . 4.00 35.00

160 Fisher Holmes. HP. Magnificent reddish scarlet .............. 4.00 35.00

300 F. J. Grootendorst. H.Pol.-Rug. Orange-red carnation-like flowers $4.50 \quad 40.00$

300 Frau Karl Druschki. HP. "White American Beauty". . . . . 4.5040 .00

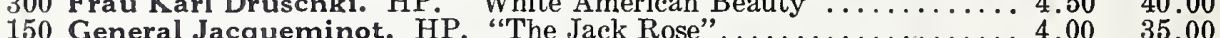

150 General Jacqueminot. H.

450 Ideal. D.Pol. Small, dark scarlet blooms borne continuously....... $4.50 \quad 40.00$

400 J. B. Clark. HP. Very large; "light red, shaded blackish maroon..... 4.00 35.00

300 Katharina Zeimet. D.Pol. White Baby Rambler" .................. $3.00 \quad 25.00$

200 Magna Charta. HP. Bright pink. One of the best in any class . . . . . 4.00 35.00

140 Margaret Dickson. HP. White, with pale rosy center.......... $4.00 \quad 35.00$

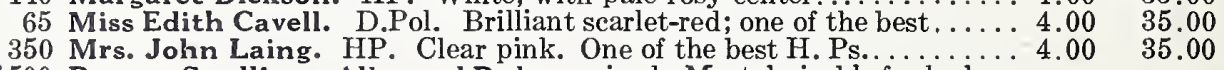

1500 Rugosa Seedlings, Alba and Rubra, mixed. Most desirable for hedges,

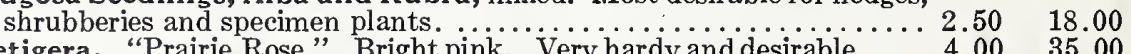

200 Setigera. "Prairie Rose." Bright pink. Very hardy and desirable.... 4.00 35.00

$800 \mathrm{Sir}$ Thomas Lipton. H.Rug. Snowy white. Fine for big specimens $3.00 \quad 25.00$

\section{For Handsome Profits, Handle Hugonis! \\ "Golden Rose of China"}

"Best hardy yellow Rose we know of, and most popular species in America."
Dr. E. H. Wilson, Arnold Arboretum

A remarkably hardy shrub Rose with clear yellow, single flowers growing on the branches like golden hollyhocks in late April or early May, Hugonis makes a symmetrical bush about 6 feet wide and as high when matured. The reddish maroon canes are in themselves most attractive and the small acacia-like foliage turns purple in the fall.

You have the chance to get all of your Hugonis here, from the most plentiful supply in America, and we feel certain you will agree, on seeing them, that they are absolutely the finest to be had, bar none. The plants are so good that you will have plenty of uses for them, for they'll make you splendid specimens as well as desirable features for the shrubbery border, the driveway, the garden background, the corner, or a handsome hedge. Plan to use a lot of them! The price this season is much less than one-tenth of the original selling-figure. That means your profits are greater, too. Better buy now!

\begin{tabular}{|c|c|c|c|c|}
\hline \multicolumn{5}{|c|}{ Vigorous dormant plants, field-grown (O.R.) } \\
\hline Extra 2 2-year... & $\ldots \ldots \ldots \ldots 60$ c. each & $\begin{array}{l}10 \\
\text { 50c. each } \\
\text { 60c. each }\end{array}$ & $\begin{array}{l}25,50,100 \\
45 \mathrm{c} \text {. each } \\
55 \mathrm{c} \text {. each }\end{array}$ & $\begin{array}{l}250,500,1,000 \text { up } \\
40 \text { c. each } \\
50 \text { c. each }\end{array}$ \\
\hline
\end{tabular}

\section{出 The Sign of America's Choicest CANNAS}

You can just as easily stock up with 4 Cannas and you'll be so much to the good. You need try just one order of novelties-your trade, we'll venture, will send you back for more. People expect the new things in this day and generation, and if you don't supply them they'll go elsewhere. You can't afford to lose a single customer but can afford to make money. Stock up with new Cannas! Order today while the assortment is complete.

Prices are for plump dormant divisions, 2 to 3 eyes, plus roots. Prices of plants from 3-in. pots, ready May 1 , are same as for dormant roots except $\$ 3$ per 100 must be added on all varieties priced below at less than $\$ 8$ per 100 .

$\begin{array}{lllll} & 10 & 100 & 1000\end{array}$

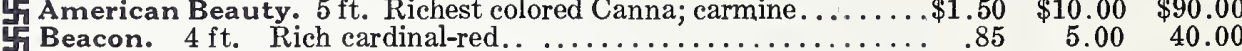

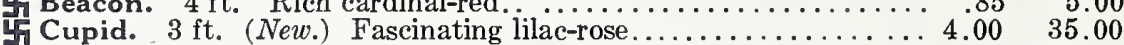
H. Edward W. Bok. (New) Soft light rose, petals edged cream....4.00 35.00 Ii Edward W. Bok. (New.) Soft light rose, petals edged cream.....4.00 45.00

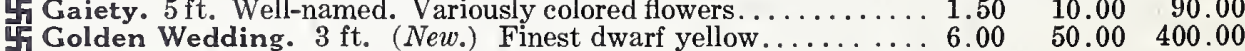
King Humbert. 4 ft. Most popular bronze-leaved variety. Flowers $6.00 \quad 50.00 \quad 400.00$

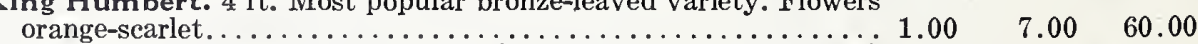
$\Psi_{\mathbf{i}}$ King Midas. $5 \mathrm{ft}$. (New.) Outstanding variety. Large flowers of

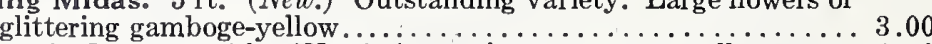

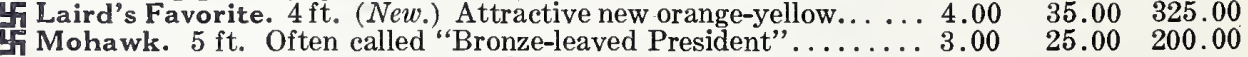

\section{Ч CANNA MRS. PIERRE S. DU PONT}

Do more with du Pont! Sell more Cannas, get more customers, make more money! Ruffled giant flowers of rich watermelon pink. Plants only, well-established in 3 in. pots: Each $\$ 1, \$ 8.50$ for $10, \$ 75$ per 100 .

4 New York. $6 \mathrm{ft}$. Carmine blooms. Bronze foliage.........\$1.00

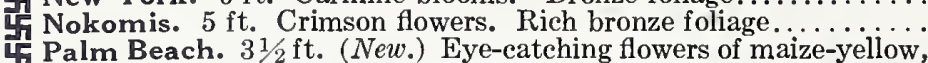
with faint blush of pink. Really rivals the finest orchids . . . . . . 3.50

Panama. $3 \mathrm{ft}$. Good bedder of rich orange red........... .85

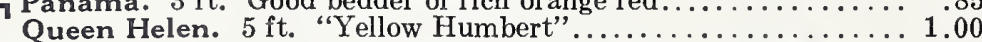

15 Razzle Dazzle. $4 \mathrm{ft}$. (New.) Dazzling crimson-maroon and yellow. 4.00 Statue of Liberty. $6 \mathrm{ft}$. Flowers blazing flame-red; leaves bronze 1.50 H Susquehanna. $3 \mathrm{ft}$. Olive green foliage. Old-rose-pink flowers. . 1.50 The Ambassador. $5 \mathrm{ft}$. A bronze-leaved President. Vigorous. . . . 4.00 4 The President. $4 \mathrm{ft}$. Far and away superior to any other red Canna 1.00 4 Victory. $3 \mathrm{ft}$. (New.) Another of the popular apricot shades..... 2.00 芹 Vulcan. 3 ft. (New.) Rich cardinal-red. Excellent to mass...... 2.50 角 Wintzer's Colossal. 5 ft. Average flowers more than cover a man's

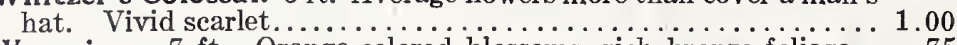
Wyoming. $7 \mathrm{ft}$. Orange-colored blossoms, rich bronze foliage.. .75 Cannas in Assortment. Good colors and selected stock....... .50

\begin{tabular}{rr}
$\$ 6.00$ & $\$ 50.00$ \\
10.00 & 90.00 \\
30.00 & 250.00 \\
5.00 & 45.00 \\
7.00 & 60.00 \\
35.00 & 300.00 \\
10.00 & 90.00 \\
12.00 & 100.00 \\
25.00 & 200.00 \\
6.00 & 50.00 \\
15.00 & 125.00 \\
20.00 & 180.00 \\
20.00 & \\
& \\
6.00 & 50.00 \\
4.00 & 35.00 \\
4.00 & 35.00 \\
\hline
\end{tabular}




\section{EVERGREENS FOR LINING OUT}

\section{Get Your Share of the Profits}

In a time when so much secondary stock is being put on the market by inexperienced growers, it is more than ever important to know your source of supply. Get this Lining-Out Stock, backed by 28 years' experience with pot grown plants for growing on. Convince yourself that here are plants rooted right, plants that will perform for you, plants that will give you the most for money! Order to-day for Spring delivery.

Twice transplanted; set out from $21 / 4$ " pots and growing in the fields since May 1926. Prices subject to change without notice.

\begin{tabular}{|c|c|c|}
\hline & & 1000 \\
\hline & $\ldots \ldots \ldots \ldots \ldots \ldots \$ 25.00$ & $\$ 225.00$ \\
\hline & Juniperus Communis Suecica (Swedish Juniper). & 225.00 \\
\hline 1 & Juniperus Sabina Tamariscifolia (Tamarix Savin) ..... & 225.00 \\
\hline & Ret. Pis. Filifera (Thread Retinospora) $\ldots \ldots \ldots \ldots \ldots \ldots \ldots \ldots$ & 180.00 \\
\hline 350 & Ret. Obtusa Compacta................ & 275.00 \\
\hline & Ret. Obtusa Gracilis (Slender Hinoki Cypress). & 275.00 \\
\hline & Ret. Pl. Lutescens (Globe form) ........... & 180.00 \\
\hline 1600 & Ret. Pisifera Aurea (Golden Sawara Retinospora)............ & 180.00 \\
\hline & 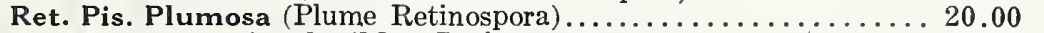 & 180.00 \\
\hline 1700 & Ret. Squarrosa Veitchi (Moss Retinospora). . & 225.00 \\
\hline 00 & Thuja Occ. Compacta (Parsons Arborvitae)... & 180.00 \\
\hline & Thuja Occ. Pumila (Green Globe Arborvitae). & 180.00 \\
\hline 200 & Thuja Occ. Siberica................... & 180.00 \\
\hline 00 & Thuja Occ. Vervaeneana (Vervaene Arborvitae).. & 180.00 \\
\hline & Thuja Occ. Vervaeneana Variegata............. & 180.00 \\
\hline & Once transplanted; growing in $2 \frac{1}{4}$ " pots since early Summer 1926. & \\
\hline & Chamaecyparis Lawsoniana Triomphe de Boskoop (Tri & 1000 \\
\hline & $\begin{array}{r}\$ 25.00 \\
12.00\end{array}$ & $\begin{array}{r}\$ 225.00 \\
100.00\end{array}$ \\
\hline & Ret. Pis. Filifera & 100.00 \\
\hline & Pisifer & 100.00 \\
\hline & awara Retinospora). & 100.00 \\
\hline & Ret. Pis. Plumosa (Plume Retinospora) ............. & 90.00 \\
\hline & Ret. Pis. Plumosa Aurea (Golden Plume Retinospora). & 90.00 \\
\hline & Ret. Squarrosa Veitchi (Moss Retinospora) ..... & 130.00 \\
\hline & Thuja Occ. Pyramidalis (American Pyramidal Arbor.). & 100.00 \\
\hline & (a) & 100.00 \\
\hline & Thuja Occ. Vervaeneana (Vervaene Arborvitae). & 90.00 \\
\hline & 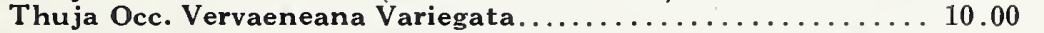 & 90.00 \\
\hline
\end{tabular}

\section{SHRUBS FOR LINING OUT}

Highly desirable pot-grown plants. 2800 Buddleia Var. Magnifica............. $11 / 4$-in. pots 2465 Buddleia Asiatica..................... pots 600 Caryopteris Incana (mastacanthus; "Blue

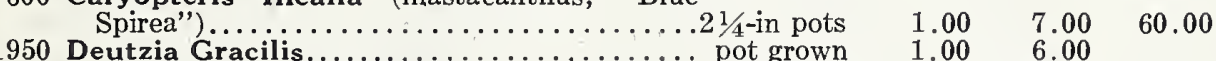
600 Deutzia Gracilis..................... pot grown $1.00 \quad 6.00$ 600 Deutzia Gracilis Rosea............... pot grown $1.00 \quad 6.00$ 1000 Deutzia Lemoinei Compacta......... pot grown 2700 Forsythia Spectabilis.................

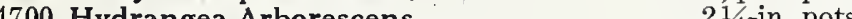
900 Lonicera Pileata............. pots 1950 Philadelphus Avalanche ............... pots 250 Philadelphus Splendens $\ldots \ldots \ldots \ldots \ldots \ldots \ldots \ldots \ldots$ 1/4 900 Philadelphus Virginal ...............

700 Spirea Anthony Waterer.............. 1300 Spirea Thunbergi................... 4000 Spirea Vanhoutte.................. 21/4-in. pots 15,000 Viburnum Tom. Plicatum(JapaneseSnowball) 3 -in. pots

$\begin{array}{rrr}10 & 100 & 1000 \\ \$ 1.00 & \$ 7.00 & \$ 60.00 \\ 1.00 & 7.50 & 60.00 \\ 1.00 & 7.00 & 60.00 \\ 1.00 & 6.00 & \\ 1.00 & 6.00 & \\ 1.50 & 12.00 & \\ 1.50 & 10.00 & 80.00 \\ 1.00 & 7.00 & 60.00 \\ 1.50 & 10.00 & \\ 1.00 & 7.00 & 60.00 \\ 1.50 & 12.00 & 100.00 \\ 1.50 & 12.00 & 100.00 \\ 1.00 & 7.00 & 60.00 \\ 1.00 & 7.00 & 60.00 \\ 1.00 & 7.00 & 60.00 \\ 2.00 & 15.00 & 125.00\end{array}$

\section{VINES FOR LINING OUT}

\section{Carefully rooted thrifty stock.}

5800 Ampelopsis Tricuspidata (Veitchi)...

$10 \quad 100 \quad 1000$

2700 Ampelopsis Tricuspidata (Veitchi), staked

plants; excellent...................

$\$ 1.50 \quad \$ 9.00 \quad \$ 75.00$

4940 Clematis Paniculata............................... pots

940 Clematis Paniculata................... pots

700 Euonymus Radicans Minimus ("Kewensis")

(Baby Wintercreeper)...............21/4-in. pots

700 Euonymus Radicans (Wintercreeper).........

700 Euonymus Radicans Variegata...........

3400 Hedera Helix (English Ivy) ..............

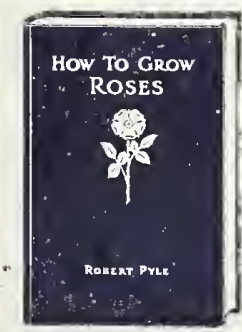

SAMPLE COPY FREE FOR 10 DAYS' EXAMINATION!

Complete, just-what-your-customers-want information on all phases of growing Roses; How to make up Rose-beds; How to Plant, Prune, Protect, etc., with a special list of varieties for various localities, etc. 192 pages chockful of meaty reading.

Price to you..................... \$1.50 per copy

Profit to you ................................

Retails at................. $\overline{\$ 2.00}$ per copy

Postage extra. Ten or more copies ordered at one time delivered free. Send for sample copy for 10 days' free examination.

For More Customers, Better-Satisfied, Try Hugonis!

(Note description on opposite page)

Vigorous dormant plants, field-grown (O. R.)

$\begin{array}{cccc}1 & 10 & 25,50,100 & 250,500,1,000\end{array}$

50......60. each 45c. each 40c. each

Extra 2 -year................. each $60 \mathrm{c}$. each $55 \mathrm{c}$. each $\quad$ 50c. each




\section{OAK BRAND SHRUBS}

Sturdy, well rooted stock, carefully graded and better than the ordinary.

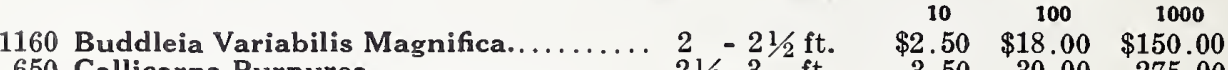
650 Callicarpa Purpurea ............... 21/2-3 $3^{\mathrm{ft}}$

1000 Caryopteris Incana (Mastacanthus). "Blue Spirea"..................... 2 ft. 320 Cornus Sanguinea............... 3 - 4 ft.

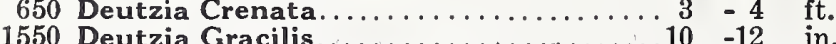

1550 Deutzia Gracilis......................

170 Deutzia Lemoinei Compacta.........18 -24 in.

720 Deutzia Crenata Magnifica............12 -18 in.

990 Deutzia Pride of Rochester........... $3=4$ ft.

900 Forsythia Spectabilis. 1 year.........

720 Forsythia Spectabilis..............

650 Forsythia Viridissima (Golden Bell)....18 -24 in

540 Hibiscus, Althea Banner............12 -15 in

2250 Hibiscus, Wm. R. Smith, 1 yr. fld.......12 -15 in.

800 Hydrangea Arborescens.................

4450 Hydrangea Panic. Grand. (Peegee)........ 12 -15 in.

1000 Hydrangea Panic. Grand. (Peegee)....... 2 - $21 / 2 \mathrm{ft}$.

380 Kerria Japonica.................. 12 -18 in.

500 Lonicera Morrowi................ 18 -24 in

500 Philadelphus Bouquet Blanc................

285 Philadelphus Manteau D'Hermine.......18 -24 in.

200 Philadel phus Splendens............ $21 / 2-3 \quad \mathrm{ft}$

3820 Philadel phus Virginal..............18 -24 in

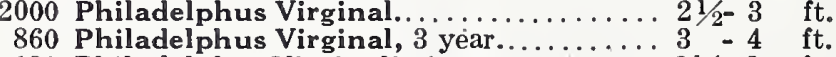

120 Philadel phus Virginalis Argentine....... $21 / 2^{-} 3 \mathrm{ft}$,

200 Philadelphus Virginalis Glacier...........

3500 Spirea Anthony Waterer, very bushy. ..... $18-24$ in

260 Spirea Prunifolia (Bridal Wreath)....... $2-2 \frac{1}{2} \mathrm{ft}$.

490 Spirea Thunbergi.................. $21 / 2-3 \mathrm{ft}$.

580 Spirea Vanhoutte...............................

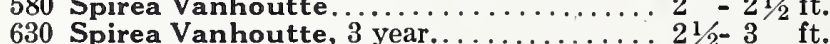

1180 Symphoricarpos Racemosus (Snowberry)

1180 Symphoricarpos Racemosus (Snowberry), 18 -24 in

460 Syringa, Lilac Persian Red............. Syringa, Vulgaris (Common Purple Lilac).18 18 in.

11,700 Viburnum Tom. Plicatum (Japanese Snowball) 1 yr.fld. (Nice mailing size).......... 9 -12 in

1500 Weigela Eva Rathke............... 18 -24 in

580 Weigela Eva Rathke...............

250 Weigela Nana Variegata, 2 year..........

\section{FIELD VINES}

400 Akebia Quinata, 2 year

\begin{tabular}{ll}
$30.00 \quad 275.00$ \\
\hline
\end{tabular}

$\begin{array}{lll}3.00 & 25.00 & 225.00\end{array}$

$\begin{array}{lll}2.50 & 20.00 & 180.00\end{array}$

$\begin{array}{lll}2.50 & 20.00 & 180.00\end{array}$

$2.00 \quad 17.50 \quad 150.00$

$\begin{array}{lll}3.50 & 30.00 & 275.00\end{array}$

$4.50 \quad 40.00$

$3.00 \quad 25.00$

$\begin{array}{lll}2.50 & 20.00 \quad 180.00\end{array}$

$\begin{array}{lll}2.00 & 15.00 & 125.00\end{array}$

$4.50 \quad 40.00$

$\begin{array}{lll}1.75 & 15.00 & 130.00\end{array}$

$\begin{array}{lll}1.75 & 15.00 & 130.00\end{array}$

$\begin{array}{lll}3.50 & 30.00 & 275.00\end{array}$

$2.75 \quad 25.00 \quad 225.00$

$\begin{array}{lll}4.00 & 35.00 & 325.00 \\ 2.00 & 16.00 & 140.00\end{array}$

$\begin{array}{lll}3.50 & 30.00 & 275.00\end{array}$

$4.00 \quad 35.00$

$2.00 \quad 15.00 \quad 125.00$

$2.50 \quad 20.00 \quad 180.00$

$2.50 \quad 20.00 \quad 180.00$

$2.50 \quad 20.00$

$4.00 \quad 35.00$

$3.50 \quad 30.00$

$5.00 \quad 45.00$

$7.00 \quad 60.00$

$7.00 \quad 60.00$

$4.50 \quad 35.00$

$7.00 \quad 60.00$

$2.50 \quad 18.00$

$3.50 \quad 30.00$

$3.50 \quad 30.00$

$2.00 \quad 18.00$

$2.50 \quad 20.00$

$2.00 \quad 15.00$

$4.00 \quad 35.00$

$2.50 \quad 20.00$

$\begin{array}{lll}3.00 & 25.00 & 180.00\end{array}$

$\begin{array}{lll}2.50 & 20.00 \quad 180.00\end{array}$

$3.50 \quad 30.00 \quad 250.00$

$\begin{array}{lll}4.50 & 40.00 & 350.00\end{array}$

$\begin{array}{lll}4.50 & 40.00 & 350.00 \\ 3.00 & 25.00 & 200.00\end{array}$

$3.00 \quad 25.00$

$\begin{array}{lll}10 & 100 & 1000\end{array}$

$\$ 2.50 \quad \$ 20.00 \quad \$ 180.00$

$\begin{array}{llll}2.00 & 18.00 & 150.00\end{array}$

$\begin{array}{lll}2.25 & 20.00 & 180.00\end{array}$

$\begin{array}{lll}3.00 & 25.00 & 225.00\end{array}$

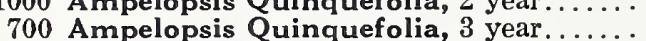

300 Celastrus Scandens (Bittersweet), $3 \mathrm{yr} .$.

1990 Clematis Paniculata, 1 yr. fld

220 Euonymus Radicans, 2 yr.

290 Euonymus Radicans Variegata, 2 yr.....

750 Lonicera Japonica Chinensis (Purple Japanese Honeysuckle)(Evergreen), 2 yr.

220 Lonicera Japonica Aureoreticulata, 2 yr.

370 Lonicera Japonica Aureoreticulata, $3 \mathrm{yr}$.

250 Lonicera Japonica Halliana, 2 yr.

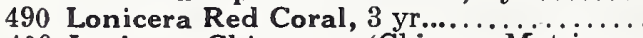

400 Lycium Chinense (Chinese Matrimony Vine), 3 yr. .

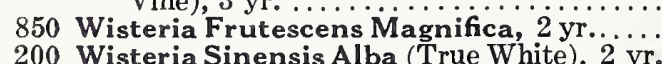

\section{HEDGE PLANTS}

10,000 Berberis Thunbergi (Japanese Barberry). 18 -24 in.

4500 Berberis Thunbergi (Japanese Barberry)..2 $\quad-21 / 2 \mathrm{ft}$.

730 Berberis Wilsonae (Wilson Barberry), 2 yr.12 -18 in.

8000 Berberis Thunbergi Minor (Box Bar-

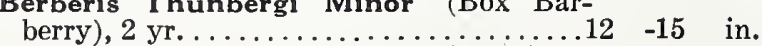

4860 Berberis Thunbergi Minor (Box Bar-

berry), 4 yr...................... 18 -24 in.

25,000
Buxus Semp. Suffruticosa (True-dwarf
Box), fid..........................

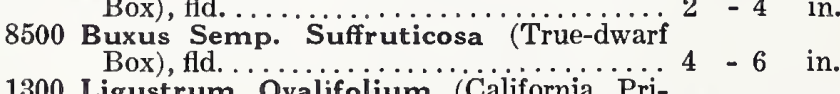

1300 Ligustrum Ovalifolium (California Pri- $21 / 2-3 \mathrm{ft}$.

1360 Ligustrum Ovalifolium (California Pri-

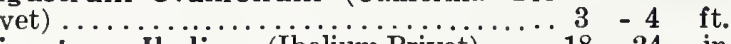

9000 Ligustrum I bolium (Ibolium Privet)..... 18 -24 in.

8000 Ligustrum Ibolium (Ibolium Privet) ..... 3 - 4 ft.

550 Ligustrum Ibota Regelianum (Regel

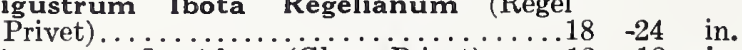

950 Ligustrum Lucidum (Glossy Privet) .................

$2.25 \quad 20.00$

2.2520 .00

$2.00 \quad 18.00$

$2.25 \quad 18.00$

$2.25 \quad 20.00$

$2.25 \quad 18.00$

$2.75 \quad 25.00$

$2.50 \quad 20.00$

$2.50 \quad 20.00$

$4.00 \quad 35.00$

$\begin{array}{lll}10 & 100 & 1000\end{array}$

$\$ 2.00 \quad \$ 15.00 \quad \$ 120.00$

$\begin{array}{lrr}3.00 & 25.00 & 220.00\end{array}$

$\begin{array}{lll}4.00 & 35.00 & 300.00\end{array}$

$2.00 \quad 16.00 \quad 140.00$

$\begin{array}{lll}3.50 & 27.50 \quad 250.00\end{array}$

$1.00 \quad 8.00 \quad 75.00$

$\begin{array}{lll}1.25 & 12.00 \quad 110.00\end{array}$

$\begin{array}{lll}.80 & 6.00 \quad 50.00\end{array}$

$\begin{array}{rrr}.90 & 7.00 & 60.00\end{array}$

$1.50 \quad 10.00$

$2.00 \quad 15.00 \quad 120.00$

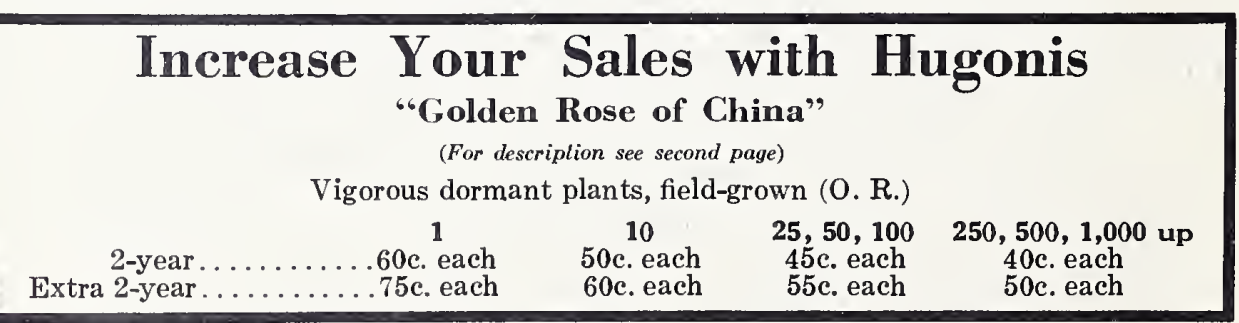

